\title{
Inmunidad colectiva contra la rubéola según una encuesta poblacional en Medellín, Colombia
}

\author{
Doracelly Hincapié Palacio, ${ }^{1}$ Juan Ospina Giraldo, ${ }^{2}$ \\ Viviana Lenis Ballesteros, ${ }^{1}$ Marta C. Ospina Ospina, ${ }^{3}$ \\ Marta Arroyave Cadavid,,$^{1}$ Nora Hoyos Muñoz ${ }^{1}$ \\ y Rita Almanza Payares ${ }^{4}$
}

Forma de citar

Hincapié Palacio D, Ospina Giraldo J, Lenis Ballesteros V, Ospina Ospina MC, Arroyave Cadavid M, Hoyos Muñoz N, Almanza Payares R. Inmunidad colectiva contra la rubéola según una encuesta poblacional en Medellín, Colombia. Rev Panam Salud Publica. 2012;32(2):101-8.

RESUMEN Objetivo. Calcular la proporción crítica $\left(P_{c}\right)$ para el logro de la inmunidad colectiva a partir de un estudio poblacional realizado en el 2009 en Medellín, Colombia, por edad, en forma global y desagregada por sexo, zona de procedencia y estrato socioeconómico.

Métodos. Se realizó una encuesta de seroprevalencia poblacional, con una muestra aleatoria de 2124 individuos de 6 a 64 años, representativa por edad, sexo y zona. Se estimó el número básico de reproducción utilizando una regresión cuadrática de los títulos promedio de IgG contra la rubéola por edad en los individuos no vacunados con títulos mayores o iguales a $15 \mathrm{UI} / \mathrm{ml}$. Se calculó el número efectivo de reproducción $\left(R_{e}\right)$ con los datos de la proporción ponderada de protección por edad, sexo, zona y estrato socioeconómico.

Resultados. En forma global, la $P_{c}$ fue de 90,0\% (IC95\% 88,6-95,2) y el $R_{e}$ de 0,95 (IC95\% 0,8-1,8), para una proporción ponderada de protección de 89,4\% (IC95\% 86,8-91,6). La protección fue menor que la $P_{c}$ esperada en ambos sexos, en los estratos socioeconómicos alto y bajo, y en la zona rural. En la zona urbana la protección fue mayor que la $P_{c}(89,4 \%$, IC95\% 86,6-91,7 en comparación con 87,4\%, IC95\% 85,2-87,8).

Conclusiones. En la zona urbana se ha avanzado hacia la inmunidad colectiva, pero se requiere aumentar la proporción de protección en forma global, en las mujeres, en la zona rural $y$ en los individuos de estrato socioeconómico alto. El número efectivo puede tener un valor mayor de uno, lo que indica el potencial de propagación de la enfermedad.

Palabras clave

Inmunidad colectiva; número básico de reproducción; rubéola (sarampión alemán); Colombia.

Universidad de Antioquia, Facultad Nacional de Salud Pública, Grupo de epidemiología, Medellín, Colombia. La correspondencia se debe enviar a Doracelly Hincapié Palacio, doracely@saludpublica.udea.edu.co

2 Universidad EAFIT, Escuela de Ciencias y Humanidades, Grupo de Lógica y Computación, Medellín, Colombia.

3 Secretaría Seccional de Salud y Protección Social de Antioquia, Laboratorio Departamental de Salud Pública, Medellín, Colombia.

4 Secretaría de Salud de Medellín, Medellín, Colombia.
El término inmunidad colectiva, poblacional o de rebaño, se refiere al efecto grupal de los programas de vacunación masiva. Se logra cuando existe una alta proporción (aunque inferior al 100\%) de individuos inmunes, lo que reduce la probabilidad de contacto entre un individuo susceptible y uno infectado, evitando la transmisión de la enfermedad (1). El término "inmunidad de rebaño" fue usado por primera vez por Topley y Wilson en 1923, que sugirieron que se separara el estudio de la inmunidad individual del de la inmunidad colectiva (2).

Para Theophil Lotz (3), cuando se vacuna no sólo se obtiene un beneficio individual, sino que este se extiende a las personas susceptibles no protegidas de una comunidad. La vacunación puede proteger a los individuos y generar un 
"cerco de protección" en torno a los susceptibles, cuando la proporción de personas inmunes es alta. Por ejemplo, si el 95\% de los individuos en una comunidad fueran inmunes a una determinada enfermedad, sería menos probable la ocurrencia de la infección por ser baja la probabilidad de contacto entre un enfermo infectante y un individuo susceptible perteneciente al 5\% existente. En caso de transmitirse la infección, no se generarían casos secundarios por estar la persona infectada en contacto primordialmente con individuos inmunes.

La inmunidad colectiva depende de la proporción de individuos inmunes y del nivel de transmisión de la enfermedad. Los indicadores utilizados para determinar el nivel de transmisión de una enfermedad son los números básico $\left(R_{0}\right)$ y efectivo $\left(R_{e}\right)$ de reproducción $(4,5) . R_{0}$ es el número promedio de casos secundarios generados por un caso índice durante su período de infecciosidad cuando este es introducido en una población completamente susceptible. $\mathrm{R}_{\mathrm{e}}$ se refiere al mismo concepto, pero cuando la población es parcialmente susceptible por la implementación de medidas de prevención y control, como la vacunación. En otras palabras, $\mathrm{R}_{0}$ refleja la transmisión de la enfermedad en el período prevacunal, $\mathrm{y}_{\mathrm{e}}$ cuando se ha instaurado la vacunación masiva (4).

Una vez que se estiman $R_{0}$ y $R_{e^{\prime}}$ se calcula la proporción mínima o crítica de individuos $\left(\mathrm{P}_{\mathrm{c}}\right)$ que deberían permanecer inmunes para el logro de la inmunidad colectiva. Para Anderson y May (5), la identificación de $\mathrm{P}_{\mathrm{C}}$ es un criterio crucial para la eliminación de una enfermedad, en cuyo caso $\mathrm{R}_{\mathrm{e}}<1$ pues, aunque se pueden generar nuevos casos, la transmisión de la enfermedad no se sostiene debido a la presencia de individuos inmunes, la reducción de la población susceptible o el incremento de las medidas de control.

En este trabajo se estimó el nivel de inmunidad colectiva contra la rubéola en Medellín, Colombia, considerando la meta de eliminación de esta enfermedad definida para las Américas para el 2010 (6).

En Colombia se inició la vacunación universal contra la rubéola en 1995 con la introducción de la vacuna contra el sarampión, la rubéola y la parotiditis (MMR) en el esquema regular de vacunación a niños de un año de edad. En 1998 se adicionó una segunda dosis a los 10 años, y en el 2002 se redujo la edad de vacunación para la segunda dosis a los cinco años. Se han realizado, además, campañas de vacunación en niños de 10 a 12 años, en jóvenes de 14 a 15 años y, en el 2005, en individuos de 14 a 39 años y en las mujeres después del parto o de un aborto (7).

La cobertura de vacunación con MMR del país ha sido superior al $90 \%$ entre el 2002 y el 2009, pero en el 2000, el 2001 y el 2011 se han obtenido coberturas inferiores al 90\%, y hay departamentos con coberturas de vacunación inferiores al 80\%. En Medellín, entre el 2005 y el 2009 se registraron coberturas con MMR superiores al 100\% debido al subregistro poblacional o a la administración de dosis de vacuna a individuos provenientes de otros lugares (8).

En este trabajo se estimó la $\mathrm{P}_{\mathrm{c}}$ de individuos inmunes por edad, en forma global y desagregada por sexo, zona y estrato socioeconómico considerando las diferencias existentes en los subgrupos de la población.

\section{MATERIALES Y MÉTODOS}

\section{Tipo de estudio}

Se calculó la proporción crítica de personas inmunes $\left(\mathrm{P}_{\mathrm{c}}\right)$ por edad, en forma global y desagregada por sexo, zona y estrato socioeconómico que se requerirían para lograr la inmunidad colectiva contra la rubéola, a partir de un estudio transversal realizado en el 2009 para determinar la seroprevalencia poblacional.

\section{Población y muestra}

El estudio se realizó en Medellín, una ciudad con 2,4 millones de habitantes distribuidos en 16 comunas de la zona urbana y 5 corregimientos de la zona rural (9). Se conformó una muestra representativa y aleatoria de 2124 individuos de 6 a 64 años, con probabilidad conocida de ser elegidos por edad, sexo y zona, de una muestra inicial de 2400 individuos. El tamaño de la muestra era suficiente para detectar una proporción de seropositividad mínima de $75 \%$, de acuerdo con los datos de un estudio de seroprevalencia de rubéola realizado en 1997 con una muestra de 864 individuos de 1 a 14 años (10).

Se utilizó un muestreo probabilístico complejo. El primer conglomerado fue la comuna o corregimiento, el segundo fue la vivienda y el tercero el hogar. La unidad final de muestreo fue la persona perteneciente al hogar. Cada unidad de muestreo se eligió mediante un muestreo aleatorio simple utilizando tablas prediseñadas de números aleatorios.

\section{Fuentes de datos}

Se aplicó una encuesta estructurada, y se efectuó toma de peso, talla y flebotomía a los individuos que no presentaban contraindicación para el procedimiento, previa firma del consentimiento informado. Se estandarizaron los procedimientos de recolección de los datos antes de efectuar el trabajo de campo. Los datos fueron recolectados por personal de enfermería con experiencia. Se solicitó a cada encuestado el carné de vacunación y la factura de servicios públicos para obtener el dato del estrato socioeconómico. El estrato bajo correspondió a la clasificación 1, 2 y 3, mientras que el estrato alto agrupó a la clasificación 4, 5 y 6 (9).

Se determinaron los títulos de IgG contra la rubéola con la prueba AxSYM ${ }^{\circledR}$ Rubella IgG (Laboratorio Abbott), y cuando los resultados estuvieron entre 5-20 UI/ml se procesaron con la prueba VIDAS RUB IgG II ${ }^{\circledR}$ (Laboratorio BioMerieux), por su mayor especificidad (11). Las muestras se procesaron en el Laboratorio Departamental de Salud Pública de la Secretaría Seccional de Salud y Protección Social de Antioquia, siguiendo un protocolo para el transporte y almacenamiento.

El proyecto fue aprobado por el Comité de Ética de la Facultad Nacional de Salud Pública. Se siguieron las disposiciones éticas para la investigación en seres humanos (12) y los principios de la Declaración de Helsinki (13).

\section{Análisis de los datos}

Se obtuvieron datos inferidos de la muestra a la población con el programa SPSS versión 15 (IBM SPSS Statistics, EE.UU.), mediante el cálculo del factor básico de expansión, esto es, el inverso de la probabilidad de ser elegido en cada comuna o corregimiento, manzana y vivienda, la ponderación de la variabilidad de cada etapa del muestreo y un factor de corrección por las diferencias en la participación por sexo (14).

La estimación del número básico de reproducción $\left(R_{0}\right)$ se hizo como una aproximación a la propuesta de Dietz 
(15) con la edad promedio de infección $(A)$ y la esperanza de vida al nacer $(L)$. En este caso, se dedujo a partir del análisis de la curva de los títulos promedio de IgG por edad. Una vez obtenido $\mathrm{R}_{0}$, se calculó la $\mathrm{P}_{\mathrm{c}}$. Los pasos para el cálculo fueron los siguientes:

1) Análisis exploratorio de los títulos promedio de anticuerpos por edad en los individuos seropositivos, es decir, aquellos con títulos de IgG contra la rubéola mayores o iguales a $15 \mathrm{UI} / \mathrm{ml}$. Se identificó el comportamiento de los títulos en forma general, en individuos vacunados y no vacunados, por la variables definidas en la muestra (edad, sexo y zona), además del estrato socioeconómico.

2) De acuerdo con la exploración, se analizó el comportamiento de los títulos mediante la función parabólica:

$$
\mathrm{Y}=\mathrm{a}+\mathrm{b}(\mathrm{c}-\mathrm{X}) \mathrm{X}
$$

donde $Y$ son los títulos promedio de anticuerpos; $X$ son los grupos de edad en intervalos trienales; $a$ es el punto de la curva donde los títulos son más bajos, por corresponder a la mitad del punto máximo; $b$ es el cambio en el nivel de anticuerpos por año de edad y $c$ es el máximo grupo de edad con títulos de anticuerpos detectables. Se estimaron los parámetros $a, b$ y $c$ mediante una regresión cuadrática con el programa NLREG $^{\circledR}$ versión 6.5 (P. Sherrod, TN, EE.UU.), a partir del ajuste de la función parabólica a los datos de seropositividad obtenidos en la encuesta.

3) Una vez estimados los parámetros, se analizó la función parabólica según se muestra en la figura 1, identificando los puntos máximos y mínimos, con el cálculo de la derivada de la función igual a cero. Este procedimiento se realizó con el programa MAPLE ${ }^{\circledR}$, versión 12 (Maplesoft Inc., Canadá). Con estos datos se estimó $A$, la edad promedio de infección en la cual los títulos promedio de anticuerpos serían la mitad del título máximo, esto es, la edad en la que probablemente ocurriría la infección en la población debido al bajo nivel de protección (5). También se estimó $L$, la edad máxima con anticuerpos detectables. Dado que los datos de la edad se encontraban agrupados en intervalos de tres años, se convirtieron en edades simples, enumerando de cero a uno los grupos de edad, donde el grupo de 6 a 8 años era la edad cero y así sucesivamente. Luego se utilizó la fórmula:

FIGURA 1. Análisis de la función parabólica $Y=a+b(c-X) X$ de la media de títulos de lgG por edad, para la estimación del número básico de reproducción; Medellín, Colombia, 2009

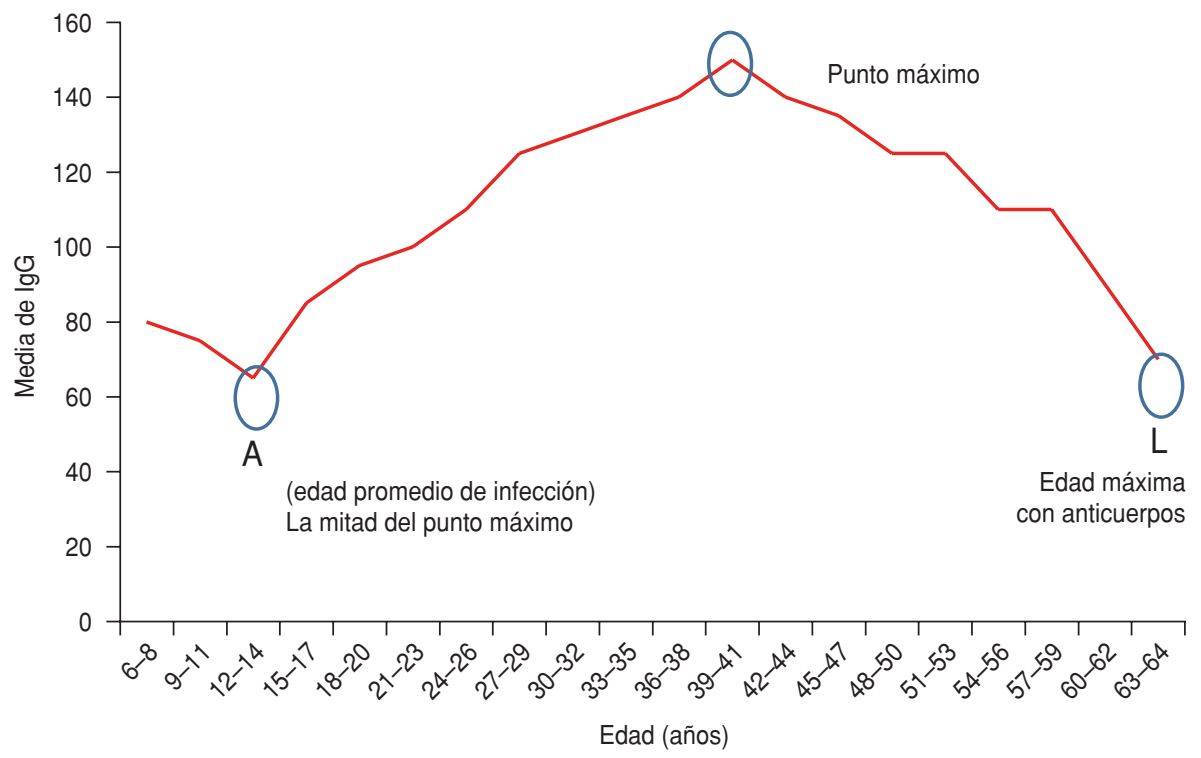

$$
x=S(X)+3 X
$$

donde $x$ es la edad simple y $X$ es el grupo de edad. $S$ es la edad superior del intervalo; por ejemplo, en el grupo de 6 a 8 años $\mathrm{S}$ toma el valor de 8.

4) Con el cálculo de $A$ y $L$ se estimó $R_{0}$, con la siguiente fórmula (5):

$$
\mathrm{R}_{0}=1+\mathrm{L} / \mathrm{A}
$$

5) Una vez calculado $R_{0}$, se estimó el número efectivo $\left(R_{\mathrm{e}}\right)$, es decir, la reducción en la transmisión ante la presencia de una cierta proporción $p$ de individuos protegidos o con inmunidad (5):

$$
\mathrm{R}_{\mathrm{e}}=\mathrm{R}_{0}(1-\mathrm{p})
$$

6) Con el cálculo de $R_{0}$ se estimó la $P_{c}$ para países en vías de desarrollo, de acuerdo con (5):

$$
\mathrm{P}_{\mathrm{c}}=\left(1-1 / \mathrm{R}_{0}\right) \times \exp (1 / \mathrm{L})
$$

7) Se obtuvieron intervalos de confianza del $95 \%$ para los números de reproducción y $\mathrm{P}_{\mathrm{c}}$ a partir de los parámetros de la regresión y la siguiente función que relaciona la edad de infección y dichos parámetros:

$$
a+b(c-X) X=\left(a+\left(b c^{2}\right) / 4\right) f
$$

donde $a+b c^{2} / 4$ es el nivel máximo de anticuerpos y $f$ es una fracción de ese nivel.

\section{RESULTADOS}

En la población estudiada, el promedio de títulos de anticuerpos IgG contra la rubéola en los individuos protegidos fue de 98,7 UI/ml (IC95\% 94,3-103,1), con una mediana de $64,5 \mathrm{UI} / \mathrm{ml}$. En este grupo habían sido vacunados contra la rubéola $40,2 \%$ ( $n=768)$, no habían recibido vacuna $32,9 \%(n=628)$ y no sabían o no se tenían datos del $26,9 \%$ restante $(n=515)$.

Con respecto al promedio de títulos de IgG por edad, en toda la población protegida se observó un comportamiento en forma de parábola, lo cual fue más evidente en los individuos no vacunados (figura 2). En los individuos no vacunados, los títulos promedio aumentaron en forma progresiva desde los 6-8 años hasta los 39-41 años, cuando se observó un pico máximo de anticuerpos, que luego descendió en forma paulatina. En los individuos vacunados el incremento de los títulos promedio desde los 6-8 años hasta los 41 años fue similar, pero a partir de esta edad los títulos mostraron un comportamiento irregular (figura 2). En algunas edades, los títulos promedio fueron inferiores en los vacunados en comparación con los no vacunados, pero la diferencia en la mediana de los títulos no fue significativa: de 15 a 17 años (prueba U de Mann-Whitney 812; $P=0,054$ ); de 21 a 23 años (U de MannWhitney 805,5; $P=0,27)$; de 45 a 47 años (U de Mann-Whitney 206; $P=0,52$ ) y de 
FIGURA 2. Media de IgG contra la rubéola en individuos protegidos ( $\geq 15 \mathrm{UI} / \mathrm{ml}$ ) según el estado de vacunación por edad; Medellín, Colombia, 2009

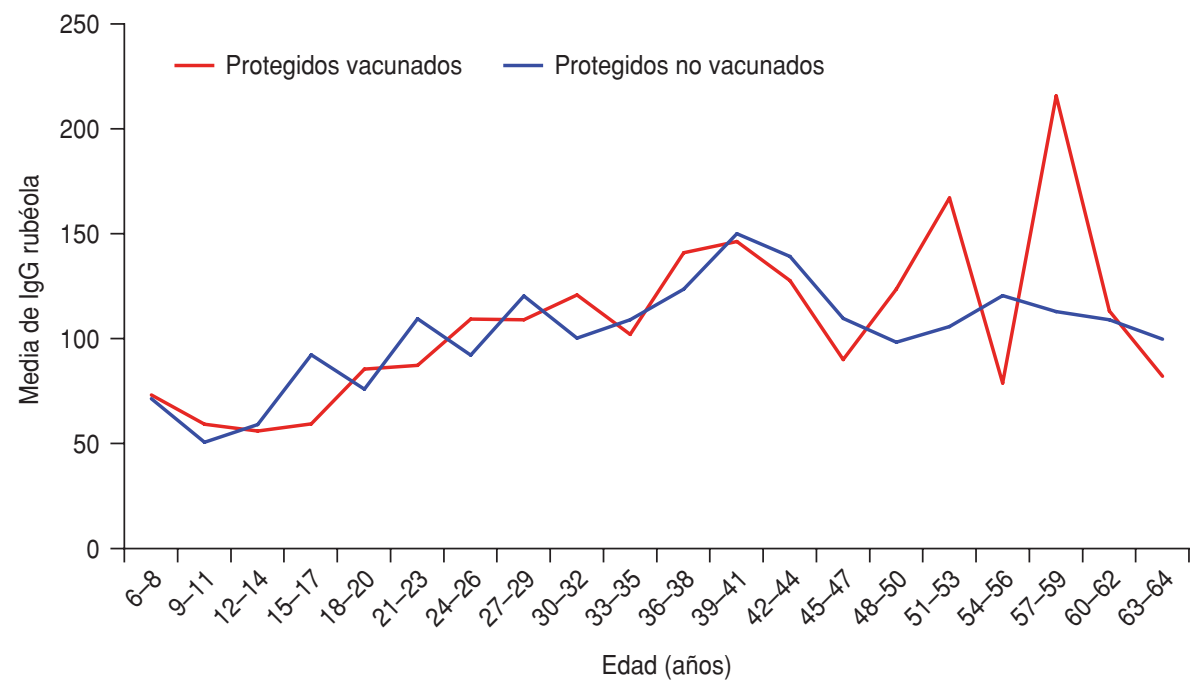

54 a 56 años (U de Mann-Whitney 166; $P=0,43)$.

Sin embargo, en el total de los individuos protegidos, independientemente de la edad, se observaron diferencias significativas en los títulos, con un promedio más alto en los no vacunados (media 86,1 ; IC95\% 79,8-92,5) que en los vacunados (media 105,8; IC95\% 98,0-113,5) (U de Mann-Whitney 203 670; $P=0,00$ ). Con respecto a los subgrupos estudiados, la forma de parábola de los títulos se repitió en individuos protegidos no vacunados, según sexo, estrato socioeconómico bajo y zona (figura 3). En los individuos de estrato socioeconómico alto se observaron títulos más altos, aunque con oscilaciones.

\section{Estimación del nivel de transmisión}

El nivel mínimo de anticuerpos por edad osciló entre 50 y $55 \mathrm{UI} / \mathrm{ml}$, excepto en la zona rural $(44,6 \mathrm{UI} / \mathrm{ml})$ y en el estrato alto $(69,0 \mathrm{UI} / \mathrm{ml})$. El aumento en los títulos de anticuerpos por edad fue mayor en la zona urbana y menor en la rural (cuadro 1).

Con respecto a la máxima edad con títulos detectables, se detectarían títulos hasta el grupo de edad número 21,5 en la zona urbana, que correspondería a 71,5 años. La mayor edad donde se detectarían títulos de anticuerpos sería en el grupo de edad número 29 de la zona rural, para una edad simple de 94 años (cuadro 1).

Para la ciudad, en el período prevacunal, $R_{0}$ estaría entre 7 y 15 casos secundarios por un caso índice (cuadro 1). Por su parte, $R_{e}$ sería menor de uno en el análisis global por edad, pero el límite superior del intervalo de confianza estaría cercano a dos. Igual situación se observó en los hombres y en los individuos de estrato socioeconómico bajo. $R_{e}$ sería ligeramente mayor de uno en las mujeres, los individuos de estrato alto y la zona rural. En la zona urbana, $\mathrm{R}_{\mathrm{e}}$ sería menor de uno y el intervalo de confianza no incluye este valor (cuadro 1).

\section{Estimación de la proporción crítica de individuos inmunes}

En forma global, para el logro de la inmunidad colectiva y la eliminación de la enfermedad la $\mathrm{P}_{\mathrm{c}}$ debería ser, al menos, de 90\% (IC95\% 88,6-95,2), la cual es superior a la proporción ponderada de protección de 89,4\% (IC95\% 86,8-91,6) (figura 4).

En los subgrupos analizados, también fue más baja la seropositividad observada $(\mathrm{Po})$ que la $\mathrm{P}_{\mathrm{c}}$ en orden de magnitud en los individuos de estrato socioeconómico alto ( $\mathrm{Po}=90,8$, IC95\% 84,1-94,8; $\mathrm{P}_{\mathrm{C}}=94,7 \%$, en las mujeres ( $\mathrm{Po}=90,3$, IC95\% 87,3-92,6; $P_{c}=91,5$, IC95\% 86,0$93,0)$, en la zona rural (Po $=89,0$, IC $95 \%$ 86,2-91,3; $P_{c}=90,2$, IC95\% 84,2-95,0), el estrato socioeconómico bajo ( $\mathrm{Po}=89,4$, IC95\% 86,5-91,8; $P_{C}=89,9$, IC95\% 86,8$94,7)$ y los hombres ( $\mathrm{Po}=88,5$, IC95\% 83,8-91,9; $P_{c}=88,6$, IC95\% 85,5-92,8). En la zona urbana fue mayor Po que $\mathrm{P}_{\mathrm{c}}$ $\left(\mathrm{Po}=89,4\right.$, IC95\% 86,6-91,7; $\mathrm{P}_{\mathrm{c}}=87,4$, IC95\% 85,2-87,8). No se pudo calcular el intervalo de confianza de la $\mathrm{P}_{\mathrm{c}}$ de los individuos de estrato socioeconómico alto, debido a la alta variabilidad de los datos observados y el inadecuado ajuste de estos a la función estimada.

\section{DISCUSIÓN}

Se estimó una $\mathrm{P}_{\mathrm{c}}$ global de $90 \%$ (IC95\% $88,6-95,2)$, superior a la proporción ponderada de protección de 89,4\% (IC95\% 86,8-91,6). Por lo tanto, si en Medellín se desea alcanzar la inmunidad colectiva contra la enfermedad se requeriría incrementar la protección hasta el $90 \%$ o más.

Dado que sólo el 10\% de los individuos son susceptibles a la rubéola, sería poco probable que se sostuvieran cadenas de transmisión, por ser alta la proporción de individuos protegidos si estos entraran en contacto con un caso en período de transmisibilidad. Sin embargo, según el valor de $R_{e}$ global, se estaría ante la eliminación de la enfermedad por ser menor de uno, pero según el intervalo de confianza se podrían generar cerca de dos casos secundarios.

Otro aspecto a considerar es la ocurrencia de fallas en la vacunación, que señalan que la $\mathrm{P}_{c}$ es un valor mínimo que debería ser superado para sostener la eliminación de la enfermedad, indicada por un $\mathrm{R}_{\mathrm{e}}<1$. De hecho, la meta de cobertura óptima de vacunación de $95 \%$ planteada para esta enfermedad (16), permitiría lograr una proporción de inmunidad o protección de entre 93 y $94 \%$, si se descuentan las fallas vacunales (17).

El objetivo de este trabajo fue mostrar la importancia de establecer no sólo "metas de vacunación e inmunidad" globales, sino también específicas por subgrupos. La proporción de individuos inmunes observada fue menor que la $P_{c}$ esperada en las mujeres y $R_{e}$ fue ligeramente mayor de uno, lo que indica la necesidad de intensificar la vacunación y el seguimiento del nivel inmunitario de las mujeres antes de la concepción (18). Igual situación se observó en los individuos de estrato socioeconómico alto y en la zona rural.

En los datos del estrato alto, se observaron oscilaciones en el promedio y la mediana de títulos de anticuerpos difícilmente captables mediante la curva parabólica u otra función, debido al tamaño reducido de los datos $(n=191)$. Sin embargo, conviene analizar las diferencias en los títulos de IgG contra la rubéola por estrato social por las diferencias en la 
FIGURA 3. Media de lgG contra la rubéola en individuos protegidos ( $\geq 15 \mathrm{UI} / \mathrm{ml}$ ) según el estado de vacunación y las variables sexo, estrato socioeconómico y zona de residencia; Medellín, Colombia, 2009
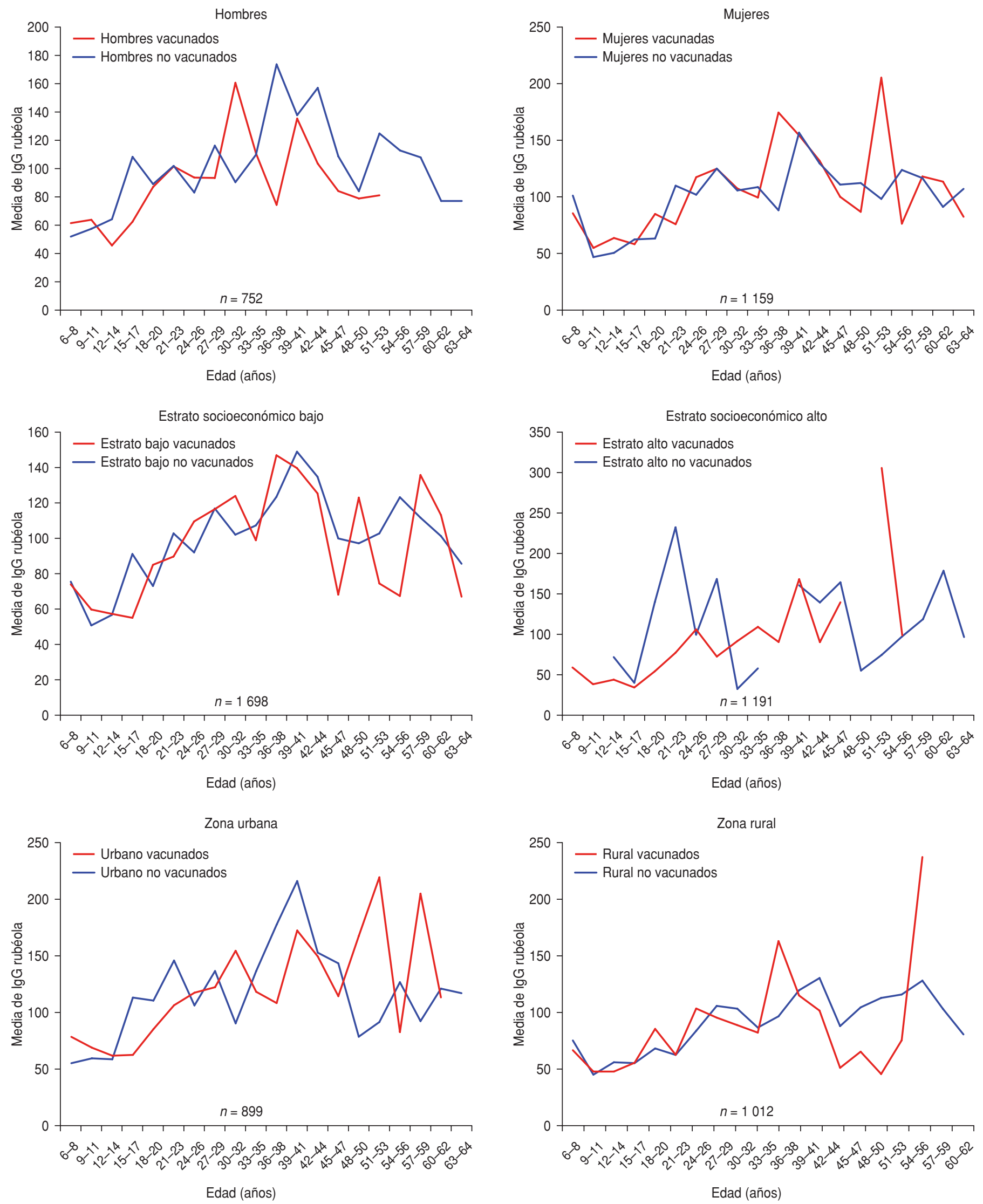
CUADRO 1. Parámetros para la estimación de los números básico y efectivo de reproducción por edad, en forma global y por subgrupos

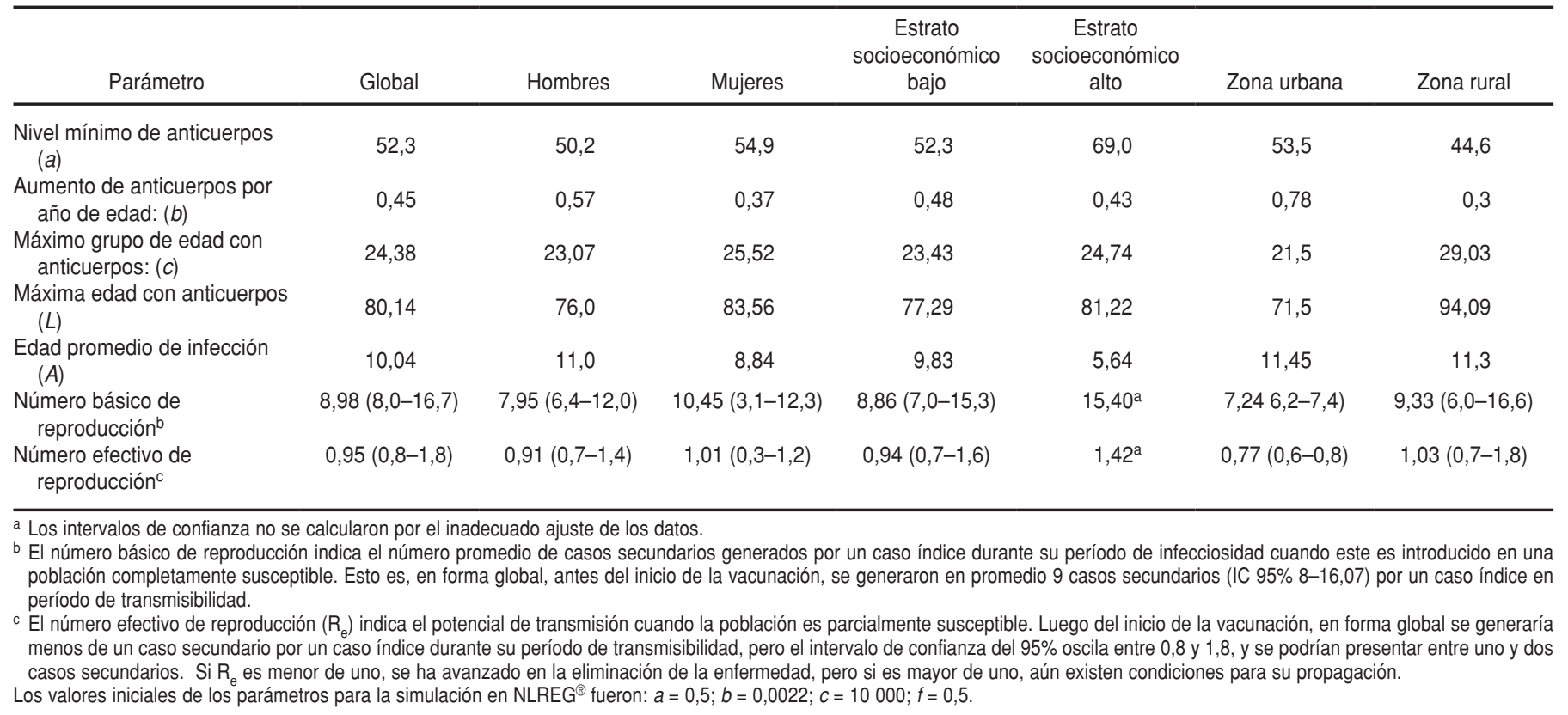

FIGURA 4. Proporción ponderada de seropositividad para rubéola y proporción crítica de individuos inmunes por edad en forma global, por sexo, estrato socioeconómico y zona; Medellín, 2009

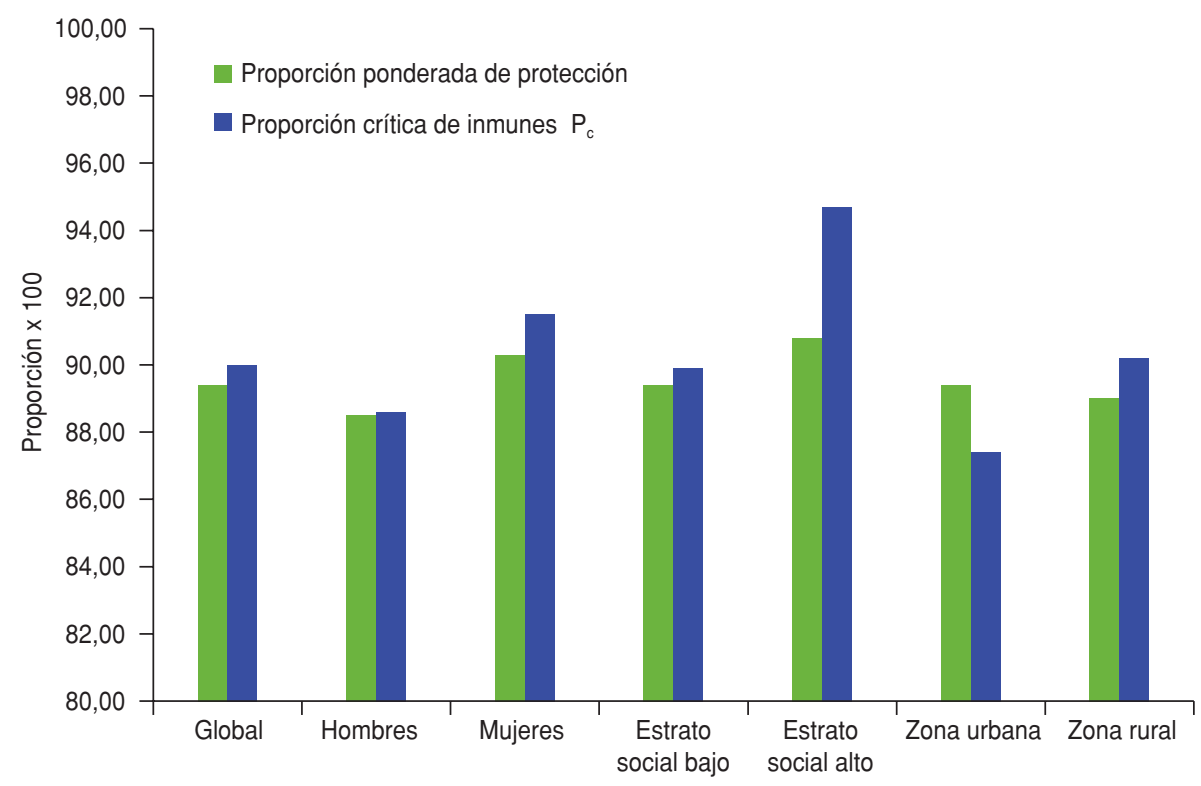

exposición al virus según las condiciones de vida $(19,20)$.

Los individuos del estrato socioeconómico alto viajaron con más frecuencia a países donde se presentaron casos en la epidemia de rubéola en las Américas entre el 2006 y el 2008 (18,9\% de individuos de estrato alto y $1,7 \%$ de estrato bajo), lo que pudo aumentar la exposición al virus (21). En los individuos de estrato bajo, la exposición podría ser mayor al requerirse un contacto prolongado, en condiciones de hacinamiento, para la transmisión de la enfermedad (18).

En la zona rural sería conveniente analizar los factores que influyen en el nivel de protección observada. Se observó una menor proporción de individuos protegidos vacunados contra la enfermedad $(36,9 \%$; tenencia de carné $73,6 \%$ ), en comparación con los de la zona urbana (43,8\%; tenencia de carné $61,7 \%)$, con una diferencia significativa $(Z=2,97 ; P=0,002)$.

En este trabajo se obtuvieron valores de $\mathrm{P}_{c}$ para la rubéola similares a otros comunicados en la bibliografía. En 1981 Dietz (15) estimó una $\mathrm{P}_{\mathrm{c}}$ de $87 \%$, correspondiente a un valor de $\mathrm{R}_{0}$ de 7,62 de acuerdo con la ecuación $R_{0}=1+L / A$. Este valor es similar al obtenido en la zona urbana, con una $\mathrm{P}_{\mathrm{c}}$ de $87,4 \%$ y un $\mathrm{R}_{0}$ de 7,24 (IC95\% 6,2-7,4). También coincide la estimación de un $\mathrm{R}_{\mathrm{e}}$ menor de uno, lo que indicaría el avance en la eliminación de la enfermedad en esta parte de la ciudad. En la misma época y con metodologías similares, Hethcote (22) calculó una $\mathrm{P}_{\mathrm{c}}$ de $87,5 \%$ y un $\mathrm{R}_{0}$ de 6,6 para Estados Unidos. Basado en este valor de $\mathrm{P}_{\mathrm{c}^{\prime}}$ Hyde (23) encontró un nivel de seropositividad global que superaba la $\mathrm{P}_{\mathrm{c}}$ tanto en el período 1988-1994 (88,1\%; IC95\% 86,9-89,1) como en el período 1999-2004 (91,3\%; IC95\% 90,5-92,1), de acuerdo con la Encuesta Nacional de Salud y Nutrición de Estados Unidos. Estos datos permiten valorar el incremento de la seropositividad tras la intensificación de la vacunación en mujeres y niños en edad escolar, aunque los autores utilizaron la $\mathrm{P}_{\mathrm{c}}$ estimada en la época prevacunal como punto de comparación. Otros autores $(17,24)$ estimaron la $P_{c} y$ sus intervalos de confianza utilizando una metodología diferente, a partir de los 
datos de laboratorios de salud pública de Inglaterra en 1986-1987. Se basaron en el supuesto de existencia de equilibrio endémico, mientras que en Medellín podría estar cercana la eliminación de la enfermedad. De acuerdo con los datos de vigilancia epidemiológica de Medellín, no se confirmaron por laboratorio casos de rubéola entre 2005 y 2011 a pesar de la búsqueda activa de casos en la comunidad e instituciones de salud. En 2011 se confirmó un caso de rubéola con fuente de infección desconocida, pero sin casos secundarios alrededor de los contactos cercanos (datos no publicados).

Una limitación del estudio fue la dificultad para verificar la vacunación, debido a la ausencia del carné en el $65,7 \%$ de los individuos encuestados. Sin embargo, en concordancia con la bibliografía, los individuos con exposición natural al virus tienen un nivel de anticuerpos más alto que los expuestos a la inmunidad artificial $(18,25)$.

Los datos de la proporción de protección fueron ponderados e inferidos de la muestra al universo; la generalización de los hallazgos puede realizarse a ciudades similares, considerando el carácter aleatorio del estudio por edad, sexo y colectiva. Vacunas. 2002;3(Supl. 2):S19-25.

2. Fine PEM. Herd immunity: history, theory, practice. Epidem Reviews. 1993;2:265-302.

3. Nishiura H, Dietz K, Eichner M. The earliest notes on the reproduction number in relation to herd immunity: Theophil Lotz and smallpox vaccination. Journ Theoret Biology. 2006;241(4):964-7.

4. Diekmann O, Heesterbeek JAP. Mathematical Epidemiology of Infectious Diseases: Model Building, Analysis and Interpretation. New York: John Wiley and Sons; 2000.

5. Anderson RM, May RM. Infectious diseases of humans: dynamics and control. New York: Oxford University Press; 1992.

6. Organización Panamericana de la Salud. Nueva meta de los programas de vacunación en la Región de las Américas: eliminar la rubéola y el síndrome de rubéola congénita. Rev Panam Salud Publica. 2003;14(5):359-63.

7. Urquijo L, Pastor D, Velandia MP, Vicari AS. Rubella and Congenital Rubella Syndrome Elimination Activities: Colombia, 2005-2006. J Infect Dis. 2011;204 (Supl 2):S603-7.

8. Ministerio de Salud y Protección Social Colombia. Coberturas de vacunación en Colombia [Internet]. Disponible en: http://www. minproteccionsocial.gov.co/salud/Paginas/ ProgramaAmpliadodeInmunizaciones(PAI). aspx Acceso el 25 de febrero de 2011. zona. Los datos por estrato socioeconómico no se pueden inferir, dado que la muestra no estuvo diseñada de acuerdo con esta variable. El tamaño de la muestra fue adecuado por la capacidad para detectar un proporción de seropositividad mínima de $75 \%$ (10).

Las estimaciones de $\mathrm{P}_{\mathrm{c}^{\prime}} \mathrm{R}_{0}$ y $\mathrm{R}_{\mathrm{e}}$ globales y por subgrupos, son guías para el reconocimiento de las diferencias logradas en el nivel de inmunidad colectiva. Fine (2) consideraba las cifras publicadas de estos indicadores globales como aproximadas y con valor básicamente comparativo por no reflejar la diversidad de las poblaciones.

En conclusión, la $P_{c}$ estimada indica el avance en el logro de la inmunidad colectiva y en la eliminación de la rubéola en la zona urbana, pero se requiere un mayor esfuerzo para mejorar y sostener el nivel de inmunidad en forma global y con énfasis en las mujeres, los individuos de estrato socioeconómico alto y de la zona rural de acuerdo con las particularidades de la población y las experiencias reportadas en la bibliografía (26). También ayudaría incorporar la estimación de $R_{e}$ en el seguimiento de la eliminación de la enfermedad (27), integrando los

\section{REFERENCIAS}

9. Departamento Administrativo Nacional de Estadística de Colombia. Estratificación Socioeconómica [Internet]. Estratificación Socioeconómica. 2012 Disponible en: http:/ / www. dane.gov.co/index.php?option=com_content \&view=article\&id=354\&Itemid=114 Acceso el 12 de febrero de 2012.

10. Rodríguez M, Díaz F, Restrepo C, Uribe G, Melguizo M, Jaramillo N. Seroepidemiología del sarampión, la hepatitis B y la rubéola en población de 1 a 14 años. Colombia Med. 1999;30(2):82-8.

11. Dimech W, Panagiotopoulos L, Francis B, Laven N, Marler J, Dickeson D et al. Evaluation of Eight Anti-Rubella Virus Immunoglobulin G Immunoassays That Report Results in International Units per Milliliter. Journ Clin Microb. 2008;46(6):1955-1960.

12. República de Colombia, Ministerio de Salud. Resolución 8430 de 1993. Bogotá, Colombia; 1993.

13. World Medical Association. Declaration of Helsinki: ethical principles for medical research involving human subjects. JAMA. 2000;284:3043-5.

14. Silva LC. Diseño razonado de muestras y captación de datos para la investigación sanitaria. Madrid: Ediciones Díaz de Santos; 2000.

15. Dietz K. The evaluation of rubella vaccination strategies. En RW Hiorns and K Cooke (dirs.). The Mathematical Theory of the Dynamics of avances de la modelación matemática en la vigilancia epidemiológica $(28,29)$, así como el seguimiento del nivel de protección conferido por la vacunación dada la menor cantidad de títulos de anticuerpos conferidos por vacunación y la menor probabilidad de circulación viral durante la eliminación (30).

Agradecimientos. Los autores agradecen la financiación del Instituto Colombiano para el Desarrollo de la Ciencia y la Tecnología COLCIENCIAS (contrato 111540820522), la Universidad de Antioquia, la Secretaría de Salud de Medellín (convenio 4600018820). También agradecen el apoyo del Laboratorio Departamental de la Secretaría Seccional de Salud y Protección Social de Antioquia; y a su equipo de trabajo: Rubén Darío Gómez A., Francisco Javier Diaz, Consuelo Lopera, Matilde Jaramillo, Morelia Cadavid, Nilton Montoya, Jader Yepes, Jhon Fredy Suárez, Cesar Higuita, Fabio Madrid, Byron Castillón, Maritza Chate, Derly Jaramillo, Luisa Fernanda Arroyave, Lina Ospina, María Eugenia Gómez, Olga Lucia Pérez, Emilse Amparo Posada, Arcene Vásquez, Gloria Tobón y Luz Marina Bernal.
Biological Populations. London: Academic Press; 198. Pp. 81-98.

16. Ministerio de la Protección Social. Lineamientos para la formulación del plan operativo anual 2011 Programa Ampliado de Inmunizaciones. [internet] Disponible en: www.minproteccionsocial.gov.co Acceso el 25 de octubre de 2011.

17. Sfikas N, Greenhalgh D, Lewis F. The Basic Reproduction Number and the Vaccination Coverage Required to Eliminate Rubella from England and Wales. Math Popul Stud. 2007;4:3-29.

18. Banatvala J, Peckham C. Rubella Viruses. Amsterdam: Elsevier; 2007. P. 139.

19. Hincapié Palacio D, Ospina Giraldo J, Gómez Arias R, Afuwape A, Chowell Puente G. Simulación del nivel de eliminación de sarampión y rubéola según la estratificación e interacción social. Rev Salud Publica (Bogotá). 2010;12(1):103-15.

20. Ospina GJ, Hincapie PD. Deterministic SIR (Susceptible-Infected-Removed) models applied to varicella outbreaks. Epidem and Infection. 2008;136:679-87.

21. Castillo-Solórzano C, Marsigli C, Bravo Alcantar P, Andrus JK, Filippis AMB, Danovaro-Holliday MC et al. Progress toward elimination of rubella and congenital rubella syndrome-The Americas 2003-2008. Centers for Diseases Control and Prevention. Morbid and Mort Weekly Report. 2008;57(43):1176-9. 
22. Hethcote, HW. Measles and rubella in the United States. Am J Epidemiol. 1983;117:2-13.

23. Hyde T, Kruszon-Moran D, McQuillan M, Cossen C, Forghani B, Reef E. Rubella immunity levels in the United States population: has the threshold of viral elimination been reached? Clin Infect Dis. 2006;43(Supl 3): S146-50.

24. Farrington $\mathrm{CP}$, Whitaker HJ. Estimation of effective reproduction numbers for infectious diseases using serological survey data. Biostatistics. 2003;4:621-32.

25. Vauloup-Fellous C, Grangeot-Keros L. Humoral Immune Response after Primary Rubella Virus Infection and after Vaccination. Clin Vaccine Immunol. 2007;14(5):644-7.
26. Castillo-Solorzano C, Marsigli C, DanovaroHolliday MC, Ruiz-Matus C, Tambini G, Andrus JK. Measles and rubella elimination initiatives in the Americas: lessons learned and best practices. J Infect Dis. 2011;204(Supl 1):S279-83.

27. Castillo-Solórzano C, Reef SE, Morice A, Andrus JK, Ruiz Matus C, Tambini G, GrossGaliano S. Guidelines for the documentation and verification of measles, rubella, and congenital rubella syndrome elimination in the region of the Americas. J Infect Dis. 2011; 204(Supl 2):S683-9.

28. De Serrés G, Gay N, Farrington P. Epidemiology of transmissible diseases after elimination. Am J Epidemiol. 2000;151(11):1039-48.
29. Orellano PW, Reynoso JI. Nuevo método para elaborar corredores endémicos. Rev Panam Salud Publica. 2011;29(5):309-14.

30. Davidkin I, Jokinen S, Broman M, Leinikki $\mathrm{P}$, Peltola H. Persistence of measles, mumps, and rubella antibodies in an MMR-vaccinated cohort: a 20-year follow-up. J Infect Dis. 2008; 197(7):950-6.

Manuscrito recibido el 17 de octubre de 2011. Aceptado para publicación, tras revisión, el 10 de junio de 2012.

ABSTRACT Objective. Calculate the critical proportion $\left(\mathrm{P}_{c}\right)$ for achieving herd immunity based on a 2009 population study conducted in Medellin, Colombia, by age, globally and disaggregated by sex, location, and socioeconomic stratum.

\section{Herd immunity against rubella according to a survey of the population in Medellin, Colombia}

Methods. A survey of seroprevalence in the population was conducted by means of a random sample of 2124 individuals aged 6 to 64 that was representative of age, sex, and location. The basic reproduction number was estimated using a quadratic regression of the average IgG titers for rubella by age in unvaccinated individuals with titers greater than or equal to $15 \mathrm{IU} / \mathrm{ml}$. The effective reproduction number $\left(\mathrm{R}_{\mathrm{e}}\right)$ was calculated with the data on the weighted proportion of protection by age, sex, location, and socioeconomic stratum.

Results. Overall, the $\mathrm{P}_{\mathrm{c}}$ was $90.0 \%$ (95\% CI, 88.6-95.2\%) and the $\mathrm{R}_{\mathrm{e}}$ was 0.95 (95\% CI, 0.8-1.8), for a weighted proportion of protection of $89.4 \%$ (95\% CI, 86.891.6\%). Protection was lower than the expected $\mathrm{P}_{\mathrm{c}}$ in both sexes, in high and low socioeconomic strata, and in the rural area. In the urban area, protection was greater than the $\mathrm{P}_{\mathrm{C}}(89.4 \%$, with a $95 \% \mathrm{CI}, 86.6-91.7 \%$, compared to $87.4 \%$ and a $95 \% \mathrm{CI}$, $85.2-87.8 \%)$.

Conclusions. The urban area has made progress toward herd immunity, but the overall proportion of protection in women, the rural area, and the high socioeconomic strata must be increased. The effective number may be greater than one, indicating the potential for the spread of the disease.

Key words Immunity, herd; basic reproduction number; rubella; Colombia. 